\title{
Emergence of Quantum Ergodicity in Rough Billiards
}

\author{
Klaus M. Frahm and Dima L. Shepelyansky* \\ Laboratoire de Physique Quantique, UMR C5626 du CNRS, Université Paul Sabatier, F-31062 Toulouse Cedex 4, France
}

(14 February 1997)

\begin{abstract}
By analytical mapping of the eigenvalue problem in rough billiards on to a band random matrix model a new regime of Wigner ergodicity is found. There the eigenstates are extended over the whole energy surface but have a strongly peaked structure. The results of numerical simulations and implications for level statistics are also discussed.
\end{abstract}

PACS numbers: 05.45.+b, 72.15.Rn, 03.65.Sq

In 1974, Shnirelman [1] proved a theorem according to which quantum eigenstates in chaotic billiards become ergodic for sufficiently high level numbers. Later it was demonstrated [2,3] that in this regime the level spacing statistics $p(s)$ is well described by random matrix theory 泊. However, one can ask the question how this quantum ergodicity emerges with increasing level number $N$ ? This question becomes especially important in the light of recent results [5,6] for diffusive billiards where the time of classical ergodicity $\tau_{D}$ due to diffusion on the energy surface is much larger than the collision time with the boundary $\tau_{b}$. In such a situation quantum localization on the energy surface may break classical ergodicity eliminating the level repulsion in $p(s)$. The investigation of rough billiards 6 showed that this change of $p(s)$ happens when the localization length $\ell$ in the angular momentum $l$-space becomes smaller than the size of the energy surface characterized by the maximal $l=l_{\max }$ at given energy $\left(\ell<l_{\max }\right)$. For $\ell>l_{\max }$ the eigenfunctions are extended over the whole surface but as we will see they are not necessarily ergodic (Fig. 1).

In this situation which we will furthermore call Wigner ergodicity the eigenstates are composed of rare strong peaks distributed on the whole energy surface. Such a case is very different from the Shnirelman ergodicity where the eigenstates are uniformly distributed. The usual scenario of ergodicity breaking was based on the image of quantum localization along the energy surface [7]. Here we show that the transition between localized and Shnirelman ergodic states can pass trough an intermediate phase of Wigner ergodicity. Our description and understanding of this phase is based on the mapping of the billiard problem with weakly rough (random) boundary on to a superimposed band random matrix (SBRM). This model is characterized by strongly fluctuating diagonal elements corresponding to a preferential basis of the unperturbed problem. Recently such type of matrices was studied in the context of the problem of particle interaction in disordered systems [8 11]. There it was found that the eigenstates can be extended over the whole matrix size while having a very peaked structure. The origin of this behavior is due to the Breit-Wigner form 12] of the local density of states according to which only unperturbed states in a small energy interval $\Gamma_{E}$ contribute to the final eigenstate.
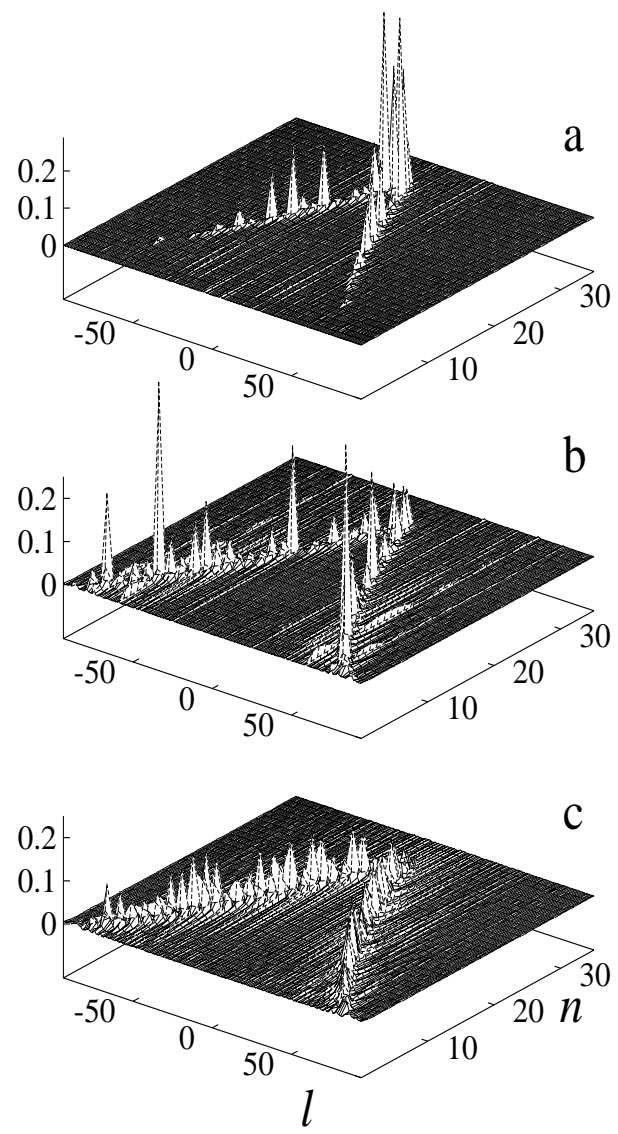

FIG. 1. Transition from localization to Shnirelman ergodicity on energy surface for level number $N \approx 2250, l_{\text {max }} \approx 95$ and $M=20$; shown are the absolute amplitudes $\left|C_{n l}^{(\alpha)}\right|$ of one eigenstate: (a) localization for $D\left(l_{r}=0\right)=20$; (b) Wigner ergodicity for $D=80$; (c) Shnirelman ergodicity for $D=1000$ (see text).

Recent optical experiments with micrometer size droplets initiated new theoretical investigations of weakly deformed circular billiards 13 . In this case the ray dynamics becomes chaotic leading to a strong directionality of light emission [14. Here we will consider another 
type of a weakly deformed circle [6], namely we chose a random elastic boundary deformation which can be represented by $R(\theta)=R_{0}+\Delta R(\theta)$ with $\Delta R(\theta) / R_{0}=$ Re $\sum_{m=2}^{M} \gamma_{m} e^{i m \theta}$ where $\gamma_{m}$ are random complex coefficients and $M$ is large but finite. This type of deformation seems to be very generic and may appear in numerous different physical situations [6]. In small droplets such boundary perturbations may be created by temperature induced surface waves. We will restrict ourself to the case of weak surface roughness given by $\kappa(\theta)=(d R / d \theta) / R_{0} \ll 1$ and all $\gamma_{m}$ being of the same order of magnitude. Then we have for the angle average $\tilde{\kappa}^{2}=\left\langle\kappa^{2}(\theta)\right\rangle_{\theta} \sim M^{2}\left(\Delta R / R_{0}\right)^{2}$.

In such a billiard the dynamics is diffusive in orbital momentum due to collisions with the rough boundary provided $\tilde{\kappa}$ is above the chaos border $\kappa_{c} \sim M^{-5 / 2}$ [6]. The diffusion constant is determined by the average change of orbital momentum per collision being $D=<(\Delta l)^{2}>=4\left(l_{\max }^{2}-l_{r}^{2}\right) \tilde{\kappa}^{2}$. This $D$ is the local diffusion rate for $l$ close to $l_{r}$. The quantum interference leads to localization of this diffusion with the length $\ell=D$ for $M<\ell<l_{\max }$ while for $\ell>l_{\max }$ the eigenstates are extended over the energy surface [6]. The transition between these two regimes is illustrated in Fig. 11. Here we present the absolute values of eigenfunction amplitudes $C_{n l}^{(\alpha)}$ in the eigenbasis $\mid n l>$ of circular billiard as a function of unperturbed radial and orbital quantum numbers $n, l$ with $\alpha$ marking the eigenenergy $E_{\alpha}$. For small roughness $\tilde{\kappa}$ (or $D$ ) the states are exponentially localized (Fig. 11a) while for large $\tilde{\kappa}$ they are homogeneously distributed (Fig. 11 c) on the energy surface. The case of Fig. 17b corresponds to an unusual regime of Wigner ergodicity where the eigenstate is extended over the surface but is composed of rare strong peaks. The positions of these peaks on the energy surface of the circular billiard $E=\mathcal{H}(n, l)$ are shown in Fig. 2a. The equation of the surface, projected on the action plane $(n, l)$, can be found from the Bohr-Sommerfeld quantization $\mu_{l}(E)=$ $\sqrt{l_{\max }^{2}-l^{2}}-l \arctan \left(l^{-1} \sqrt{l_{\max }^{2}-l^{2}}\right)+\pi / 4=\pi(n+1)$ where $l_{\text {max }}^{2}=4 N=2 m R_{0}^{2} E / \hbar^{2}=k^{2} R_{0}^{2}$ with $k$ being the wave number. A part of the surface is shown in more detail in Fig. 2fb. Here it is clearly seen that the peaks are large for those integer $n, l$ which are close to the line $\mathcal{H}(n, l)=E_{\alpha}$. Our understanding of the fact that not all integer values of the $(n, l)$-lattice near to this line are populated is based on the concept of Breit-Wigner structure of eigenstates described below.

According to Refs. 66, 15 the internal scattering at the rough boundary can be described by the $S$-matrix

$$
S_{l \tilde{l}}(E)=e^{i \mu_{l}(E)}<l\left|e^{i V(\theta)}\right| \tilde{l}>e^{i \mu_{\tilde{l}}(E)},
$$

with $\mu_{l}(E)$ being the scattering phases of the circle and $V(\theta)=2 \sqrt{l_{\max }^{2}-l_{r}^{2}} \Delta R(\theta) / R_{0}$. This quantum rough map [6] is defined with respect to amplitudes $a_{l}$ in the wave function expansion $\psi(r, \theta)=B \sum_{l} a_{l} J_{|l|}(k r) e^{i l \theta}$ with Bessel functions $J_{l}$ and $B$ being a normalization constant. The $S$-matrix gives a local unitary description for $l$ close to a resonant value $l_{r}$. The eigenvalue equation reads $\sum_{\tilde{l}} S_{l, \tilde{l}}\left(E_{\alpha}\right) a_{\tilde{l}}^{(\alpha)}=a_{l}^{(\alpha)}$ so that the eigenvalues $E_{\alpha}$ are determined by $\operatorname{det}\left[1-S\left(E_{\alpha}\right)\right]=0$. For $V=0$, we recover the Bohr-Sommerfeld quantization for eigenvalues $E_{n l}$ of the ideal circle.

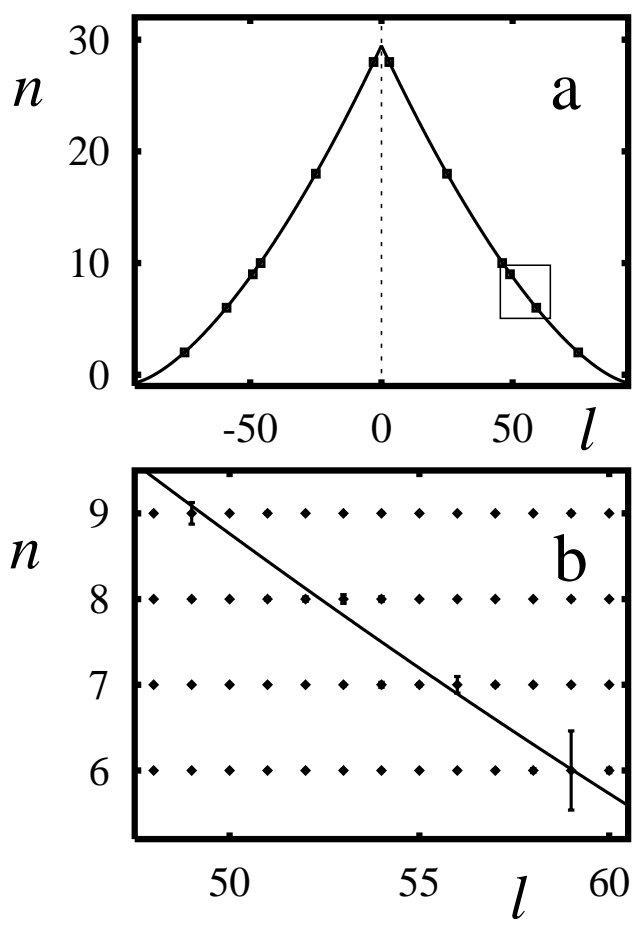

FIG. 2. (a) Main peaks of eigenstate in Fig. 1b (squares for $\left.\left|C_{n l}^{(\alpha)}\right| \geq 0.1\right)$ shown on the energy surface $\mathcal{H}(n, l)=E_{\alpha}$ (see text); (b) rescaled part of (a): diamonds show the integer $(n, l)$-lattice, the errorbar size is $2\left|C_{n l}^{(\alpha)}\right|$.

The semiclassical regime of ray dynamics corresponds to the limit $V \gg 1$ where the $\theta$-integral can be evaluated in a saddle point approximation giving the classical limit of quantum rough map [6. Here we are interested in a different regime where $V<1$ corresponding to $D<M^{2}$. There, by the mapping on an effective solid state Hamiltonian $H_{\text {eff }}$ introduced by Fishman, Grempel, and Prange [16], the equation for eigenstates takes the form

$$
\tan \left[\mu_{l}\left(E_{\alpha}\right)\right] a_{l}^{(\alpha)}+\frac{1}{2} \sum_{\tilde{l}}<l|V| \tilde{l}>a_{\tilde{l}}^{(\alpha)}=0 .
$$

In this way the eigenvalue equation is reduced to a solid state problem with $2 M$ coupled sites. The $H_{\text {eff }}$-matrix is of SBRM type with strongly fluctuating diagonal elements produced by scattering phases $\mu_{l}$. The investigations of such matrices [9-11] showed that the local density of states has the Breit-Wigner width given by the Fermi golden rule $\Gamma_{\mu}=2 \pi \rho_{\mu}<(V(\theta) / 2)^{2}>\approx 3 D /\left(2 M^{2}\right)$ where $\rho_{\mu}=1 / \pi$ is the density of diagonal elements and 
we used the relation between phase average of $V^{2}(\theta)$ and $D$. This expression is valid [17] when $\Gamma_{\mu}$ exceeds the mean level spacing $(\sim 1 / M)$ in the band width $M$. In the opposite limit $\Gamma_{\mu} M<1$ the eigenstates are given by standard perturbation theory. Together with the condition $V<1$ we find that the Breit-Wigner regime exists for $M<D<M^{2}$ near zero energy of $H_{e f f}$. In this regime the localization length is $\ell=D$ [6, 8 11, 15]. However, the Breit-Wigner structure remains in both localized $\left(\ell<l_{\max }\right)$ and delocalized $\left(\ell>l_{\max }\right)$ cases if $M<D<M^{2}$. Therefore for $l_{\max }<D<M^{2}$ the states are extended but only $l$ with $\left|\tan \left(\mu_{l}\right)\right|<\Gamma_{\mu}<1$ are mixed leading to a peaked structure of eigenstates 18 . The fraction of peaks in $\max \left(\ell, l_{\max }\right)$ is $\Gamma_{\mu}$.

The above properties of scattering amplitudes $a_{l}^{(\alpha)}$ allow to understand the behavior of eigenfunction coefficients $C_{n l}^{(\alpha)}=<\psi_{\alpha} \mid n l>$. For this one has to compute the expansion $J_{l}\left(k_{\alpha} r\right) e^{i l \theta}$ in terms of $\mid n l>$. Since $\Delta R \ll R_{0}$ the angular and radial integrals factorize and can be evaluated using the radial eigenvalue equation and the semiclassical expression for $J_{l}(k r)$. As a result we obtain

$$
C_{n l}^{(\alpha)} \approx \tilde{B} a_{l}^{(\alpha)}\left(l_{\max }^{2}-l^{2}\right)^{1 / 4} \frac{\sin \Delta \mu}{\Delta \mu},
$$

with $\Delta \mu=\mu_{l}\left(E_{\alpha}\right)-\mu_{l}\left(E_{n l}\right) \approx\left(E_{\alpha}-E_{n l}\right) / E_{b}$ and $E_{b}=d E / d \mu_{l}(E)=\hbar^{2} l_{\max }^{2} /\left(m R_{0}^{2} \sqrt{l_{\max }^{2}-l^{2}}\right)=2 \hbar / \tau_{b}$ being the energy scale related to the ballistic collision time $\tau_{b}, \tilde{B}$ is a normalization constant. The amplitudes $C_{n l}^{(\alpha)}$ determine the local density of states by

$$
\rho_{W}\left(E-E_{n l}\right)=\left\langle\sum_{\alpha} \delta\left(E-E_{\alpha}\right)\left|C_{n l}^{(\alpha)}\right|^{2}\right\rangle
$$

The averaging is performed with respect to different roughness realizations and/or over a sufficiently large energy interval. Due to the Breit-Wigner distribution for $\tan \left(\mu_{l}\right)$ in (2) we obtain

$$
\rho_{B W}\left(E-E_{n l}\right)=\frac{1}{\pi} \frac{\Gamma_{E} / 2}{\left(E-E_{n l}\right)^{2}+\Gamma_{E}^{2} / 4}
$$

with

$$
\Gamma_{E}=E_{b} \Gamma_{\mu}=E_{b} \frac{N}{N_{W}}\left(1-\frac{l^{2}}{l_{\max }^{2}}\right), N_{W}=\frac{M^{2}}{24 \tilde{\kappa}^{2}} .
$$

The equations (5), (6) are valid for $\Delta E<E_{B}(\Delta \mu<1)$ and $\Gamma_{\mu}<1$ or $N<N_{W}$. Here $N_{W}$ is the border of Breit-Wigner regime in level number $N$. We remind that the eigenstates are localized for $N<N_{e}=1 /\left(64 \tilde{\kappa}^{4}\right)$ corresponding to $\ell<l_{\max }$ [6]. As a result the Breit-Wigner structure can exist both in the localized and delocalized cases. An example of Breit-Wigner distribution is shown in Fig. 3. Our numerical data confirm the theoretical expression (6) for variation of $\Gamma_{\mu}$ by more than one order of magnitude (inset).

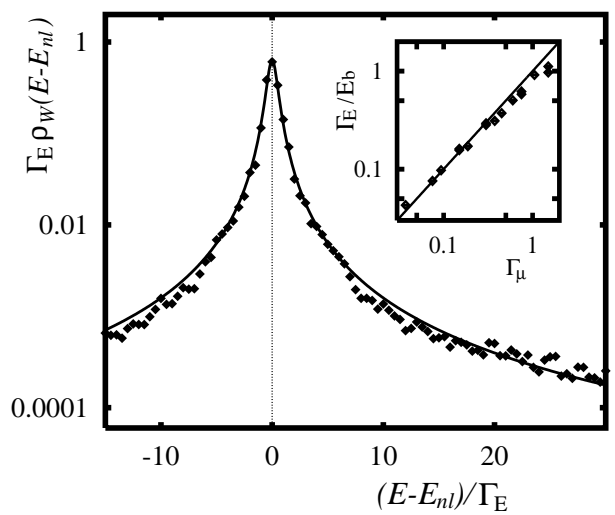

FIG. 3. Breit-Wigner distribution for eigenstates of rough billiard (diamonds) with the parameters of Fig. 1b (5 eigenstates for each of 10 roughness realizations are used). Full curve gives the distribution (5) with the theoretical $\Gamma_{E}$ value (6), $\Gamma_{E} / E_{b}=\Gamma_{\mu}=0.3$. Insert shows the variation of $\Gamma_{E} / E_{b}$ (diamonds) as a function of $\Gamma_{\mu}$ for parameter range $10 \leq M \leq 40$ and $M \leq D \leq M^{2}\left(E_{b}\right.$ and $\Gamma_{\mu}$ are taken at $l=0)$. The theory $(\overline{6})$ is shown by straight line.

For $N>N_{W}$ the kick amplitude $V$ in (1) is larger than 1 and the mapping on to equation (2) is not valid. In this case the scattering phases (eigenphases of $S$ ) are homogeneously distributed in the interval $(0,2 \pi)$. If in addition $N>N_{e}$ then as in the case of kicked rotator (see B. V. Chirikov in [3]) the amplitudes $a_{l}$ are homogeneous in $l$-space with $\left|a_{l}\right|^{2} \approx 1 /\left(2 l_{\max }\right)$. Using Eq. (3), we obtain that the local density of states is given by

$$
\rho_{W}\left(E-E_{n l}\right)=\frac{E_{b}}{\pi} \frac{\sin ^{2}\left[\left(E-E_{n l}\right) / E_{b}\right]}{\left(E-E_{n l}\right)^{2}} .
$$

This density is normalized to one and as a result the probability $\left|C_{n l}^{(\alpha)}\right|^{2}$ is ergodically distributed along the energy surface shown in Fig. 2a. This is the regime of Shnirelman ergodicity which emerges for $N>$ $\max \left(N_{W}, N_{e}\right)$. For fixed roughness $\tilde{\kappa}>\kappa_{E W}=\sqrt{6} / 4 M$ we have $N_{W}>N_{e}$ and the transition to Shnirelman ergodicity with the increasing level number $N$ crosses the region of Wigner ergodicity for which an eigenfunction is ergodic only inside the Breit-Wigner width $\Gamma_{E}<E_{b}$. In the opposite case $\kappa_{c}<\tilde{\kappa}<\kappa_{E W}$ the Shnirelman ergodicity emerges directly from the localized phase (the BreitWigner regime exists only in the localized phase). The averaging of equation (7) over different $l$-values gives the distribution (5) with $\Gamma_{E} \sim E_{b}$. This explains why also for the case $\Gamma_{\mu}<1$ in Fig. 3 the distribution (5) remains valid even for $\Delta E>E_{b}$ (note that $\pi E_{b} / \Gamma_{E} \approx 10$ ).

The above analysis shows that in the regime of Wigner ergodicity there are four relevant energy scales: level spacing $\Delta$, Thouless energy for diffusion in $l$-space $E_{c}=$ $\hbar D /\left(l_{\text {max }}^{2} \tau_{b}\right)$, the Breit-Wigner width $\Gamma_{E}=3 \hbar D /\left(M^{2} \tau_{b}\right)$ and bouncing energy $E_{b}=2 \hbar / \tau_{b}$ which are ordered as $\Delta<E_{c}<\Gamma_{E}<E_{b}$. These scales should appear in the level statistics namely for the number variance 
$\Sigma_{2}(E)$ [3]. For $E<E_{c}$ as usual we expect gaussian orthogonal ensemble (GOE) statistics to be valid while in the interval $E_{c}<E<\Gamma_{E} / 2$ the behavior should be modified, due to the diffusive dynamics [19], being $\Sigma_{2}(E) \sim\left(E / E_{c}\right)^{1 / 2}$. The first investigations of the regime with $\Gamma_{E} / 2<E<E_{b} / 2$ for SBRM were done only recently 20. They showed that level rigidity is strongly suppressed with a nearly linear energy behavior in $\Sigma_{2}(E)$ due to disappearance of correlations between levels with energy differences larger than $\Gamma_{E}$. However, such local characteristics as $p(s)$ are still described by GOE if $E_{c} \gg \Delta$. Our numerical data qualitatively confirm these expectations (see Fig. (4), however, quantitative numerical and analytical verifications are still required. In Fig. 4 1 the above energy scales are not separated by strong inequalities but parametrically it is possible to have them. In this unusual regime it would be interesting to study other physical properties. We mention for example the frequency dependence of dielectrical response [21] which should be sensitive to the above energy scales.

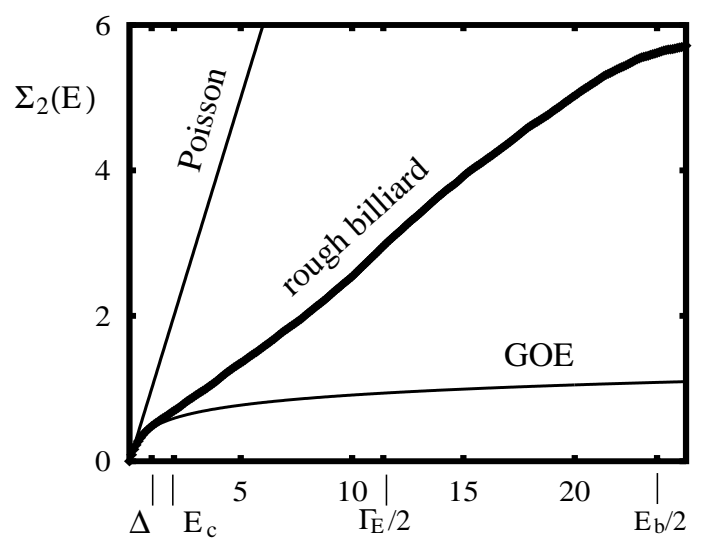

FIG. 4. Dependence of number variance $\Sigma_{2}(E)$ on energy for rough billiard compared to Poisson and GOE for $M=50$, $D=800, l_{\max } \approx 95 ; 6$ roughness realizations in the interval $2150<N<2350$ are used for the average. The energy scales are also shown in units of level spacing $\Delta$.

In conclusion, we studied the parameter dependence of the quantum energy surface width in rough billiards. In the limiting case of Shnirelman ergodicity with high level numbers, this width is determined by the typical frequency of collisions with the boundary $\left(\Gamma_{E} \sim E_{b}\right)$. This means that all integer points on the $(n, l)$-lattice of quantum numbers with a distance $\Delta l=\Delta n \approx 1$ from the energy line $E_{\alpha}=\mathcal{H}(n, l)$ are occupied by one eigenfunction $\psi_{\alpha}\left(N>N_{W}\right.$ and $\left.N>N_{e}\right)$. We have found a new regime of Wigner ergodicity where $\Gamma_{E} \ll E_{b}$ so that only points with $\Delta l=\Delta n \leq \Gamma_{\mu} \ll 1$ contribute to $\psi_{\alpha}$. As a result a lot of holes appear in the energy surface and $\psi_{\alpha}$ has a strongly peaked structure on $(n, l)$-lattice. However, at the same time all states in the Breit-Wigner width $\Gamma_{E}$ are populated so that the eigenfunction is ergodic inside this energy band. It would be interesting to understand if the arithmetical properties of the lattice will play an important role.

* Also Budker Institute of Nuclear Physics, 630090 Novosibirsk, Russia

[1] A. I. Shnirelman, Usp. Mat. Nauk. 29, N6, 18 (1974).

[2] O. Bohigas, M.-J. Giannoni, and C.Schmit, Phys. Rev. Lett. 52, 1 (1984); O. Bohigas in [3.

[3] Les Houches Lecture Series 52, Eds. M.-J. Giannoni, A.Voros, and J. Zinn-Justin (North-Holland, Amsterdam, 1991).

[4] M.L. Mehta, Random Matrices (Academic Press, New York, 1991).

[5] F. Borgonovi, G. Casati, and B. Li, Phys. Rev. Lett. 77, 4744 (1996).

[6] K. M. Frahm and D. L. Shepelyansky, Phys. Rev. Lett. 78, 1440 (1997).

[7] G. Casati, B. V. Chirikov, I. Guarneri and F. M. Izrailev, Phys. Rev. E 48, 1613 (1993); Phys. Lett. A 223, 430 (1996).

[8] D. L. Shepelyansky, Phys. Rev. Lett. 73, 2607 (1994).

[9] P. Jacquod and D. L. Shepelyansky, Phys. Rev. Lett. 75, 3501 (1995).

[10] Y. V. Fyodorov and A. D. Mirlin, Phys. Rev. B 52, R11580 (1995).

[11] K. Frahm and A. Müller-Groeling, Europhys. Lett. 32, 385 (1995).

[12] E. P. Wigner, Ann. Math. 62, 548 (1955); 65, 203 (1957).

[13] A. Mekis, J. U. Nöckel, G. Chen, A. D. Stone, and R. K. Chang, Phys. Rev. Lett. 75, 2682 (1995).

[14] J. U. Nöckel and A. D. Stone, Nature 385, 45 (1997).

[15] K. M. Frahm, Phys. Rev. B (to appear).

[16] S. Fishman, D. R. Grempel, and R. E. Prange, Phys. Rev. Lett. 49, 509 (1982).

[17] The situation here is a bit different from 19 11 due to translational invariance of $\langle l|V| \tilde{l}\rangle$ in $l$. But this does not change the result which can be derived exactly for random phases $\mu_{l}$ using the Lloyd model fluctuations.

[18] Note that for the case studied in [6] with $\gamma_{m} \sim 1 / m$ this Breit-Wigner regime does not exist, since there $D \sim$ $M\left\langle V^{2}\right\rangle$ instead of $D \sim M^{2}\left\langle V^{2}\right\rangle$ for $\gamma_{m} \sim$ const.

[19] B. L. Al'tshuler and B. I. Shklovskii, Zh. Eksp. Teor. Fiz. 91, 220 (1986) [Sov. Phys. - JETP 64, 127 (1986)].

[20] D. Weinmann and J.-L. Pichard, Phys. Rev. Lett. 77, 1556 (1996).

[21] L. P. Gor'kov and G. M. Eliashberg, Zh. Eksp. Teor. Fiz. 48, 1407 (1965) [Sov. Phys. - JETP 21, 940 (1965)]. 